\title{
Design and Evaluation of MAC Protocol Strategies Techniques in Wireless Ad Hoc Network
}

\author{
Prince Kumar $^{1}$, Rashmi Raj ${ }^{2}$ \\ ${ }^{1}$ M.Tech Scholar, ${ }^{2}$ Assistant Professor, \\ Department of Electronics and Communication Engineering, \\ Universal Institute of Engineering, Technology, Mohali, Punjab, India
}

\begin{abstract}
Wireless communication has become a core part of modern communication technology. The Infrastructure less wireless network, commonly referred to as Ad Hoc networks, has attracted extensive research interest for past 30 years. In this work, the focus is on IEEE 802.11 network performance analysis of Multihop hop Ad Hoc networks under non-saturated network conditions. To meet the increasing demand of multimedia, it is necessary to provide the quality of service in such networks. The current work presents the development of an analytical model for network performance analysis.
\end{abstract}

The medium access mechanism in multihop wireless networks should minimize collisions, and take care of the hidden and exposed node problems. The IEEE 802.11 MAC with Distributed Coordination Function (DCF) does not scale well in such networks. We introduce Point Coordination Function (PCF) in the region of high traffic areas, and discuss its effect on network performance. To improve network scalability and throughput, we propose the design of a new MAC called Dual MAC. This work discusses architecture and working of the dual MAC in detail. Performance results of the network using dual MAC are presented, and compared with that of pure DCF operation.

Keywords: Wireless Communication, MAC Protocol, Multihop ad-hoc wireless networks

\section{INTRODUCTION}

In recent times, the wireless networks have become very popular. Wireless LANs are being deployed on airports, conferences, etc. People have started using portable laptops to access Internet and other resources using wireless networks while moving. Another area which has generated a lot of interest recently, is wireless ad- hoc networks. An ad-hoc network is formed when two or more stations come together form an independent network. Ad-hoc networks are also termed as infrastructure-less networks since as they do not require any prior infrastructure. Two stations that are within transmission range of each other are called one hop neighbors. Multihop ad-hoc networks are ones in which the stations can talk to stations more than one hop away via intermediate stations. Cooperative ad-hoc networks are formed by several homogeneous wireless stations. All the stations cooperate with each other, i.e., the traffic for the stations that are more than one hop away is routed by the intermediate stations.

The intermediate stations are called relaying stations. The following section describes the common Media Access Control layer used by the 802.11 family of standards. The 802.11 family uses a MAC layer known as CSMA/CA (Carrier Sense Multiple Access/Collision Avoidance) NOTE: Classic Ethernet uses CSMA/CD - collision detection). CSMA/CA is, like all Ethernet protocols, peer-to-peer (there is no requirement for a master station).The basic transmission process suffers from the hidden terminal problem and the exposed terminal problem. To mitigate the above problem additional control packets, request-to-send and clear-to-send control frames, are transmitted prior to the exchange of the actual data frame. A successful exchange of these control packets ensures that channel is reserved for the time period enough to complete the data transfer process. 


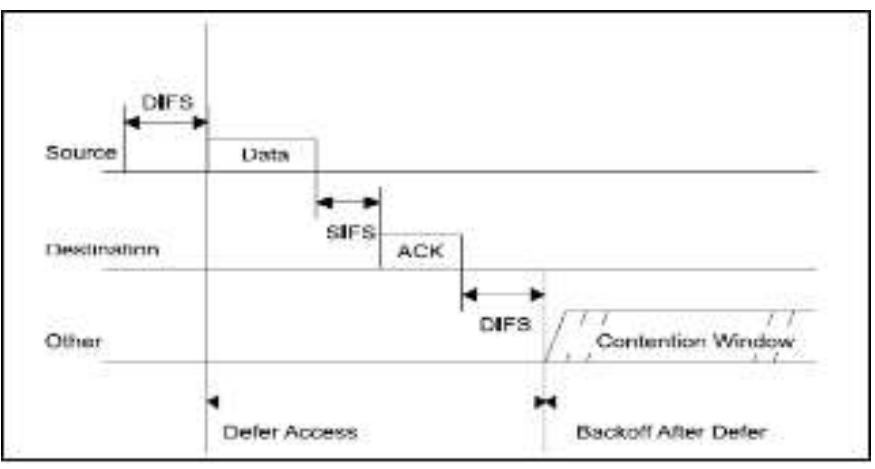

Figure 1: Basic Mech for data exchange Between two nodes

\section{Original 802.11 Mac DCF}

The DCF is the fundamental access method used to support asynchronous data transfer on a best effort basis. The DCF is based on CSMA/CA. The carrier sense is performed at both the air interface, referred to as physical carrier sensing, and at the MAC sub layer, referred to as virtual carrier sensing. Physical carrier sensing detects presence of other users by analyzing the activity in the channel through the received signal strength. A station performs virtual carrier sense by examining the received MPDU (MAC Protocol Data Unit) information in the header of RTS, CTS and ACK frames. The stations in BSS use this information to adjust their Network Allocation Vector (NAV), which indicates amount of time that must elapse until the current transmission is complete and the channel can be sampled again for idle status. Priority access to the medium is controlled through the use of mandatory inter frame space (IFS) time intervals between the transmissions of frames. Three IFS intervals are specified in the standard: Short IFS (SIFS), PCF-IFS (PIFS), and DCF-ISF (DISF).

\section{PCF}

The 802.11 MAC offers contention free service by means of PCF. PCF is optional capability and provides contention-free $(\mathrm{CF})$ frame transfers. The PCF relies on the point coordinator (PC) to poll other stations. The polled stations can send the data without contending for the medium. In a wireless LAN, the function of a PC is performed by AP within each $\mathrm{BSS}$. The PCF is required to coexist with the DCF and logically sits on the top of DCF (see Figure 3.4). The PCF consists of alternating contention free period (CFP), and contention period (CP) as shown in figure 3.6. In the CFP, the PC polls each of the stations present in the BSS. The PC specifies the start of the CFP by sending a beacon that contains the length of CFP duration, among other things. All the stations in the BSS set their NAV for the duration of the CFP. The PC terminates the CFP by sending a CF-End frame, and may also terminate it before the advertised CFP duration. The time difference between two beacons is called beacon period (BP) or CFP repetition interval, and is a multiple of beacon frame. The beacon also helps in synchronization and timing. The limits on durations of each of the frames are described in the IEEE 802.11 standard [5]. In the CP, the stations use DCF to access the medium.

\section{Ieee 802.11 Mac Protocol Operation}

The IEEE 802.11 MAC offers two kinds of medium access methods, namely Distributed Coordination Function (DCF), and Point Coordination Function (PCF). DCF is the basic access method in 802.11 and requires no infrastructure. When wireless stations are within transmit range of each other, they form a Basic Service Set (BSS), and can communicate to each other using DCF. If the BSS contains only two stations, it is called Independent Basic Service Set (IBSS). Many BSSs may be connected by a Distribution System (DS) to form an Extended Service Set (ESS). An access point (AP) is the station that provides access to DS services. The PCF is built on the top of the DCF, and is also referred to as infrastructure mode. It requires a polling station called Point Coordinator (PC), which acts as controlling station during poll. The PCF consists of alternating Contention Free Periods (CFP) and Contention Periods (CP). During CFP, the PC polls other stations in the medium, and during $\mathrm{CP}$, the access method becomes DCF.

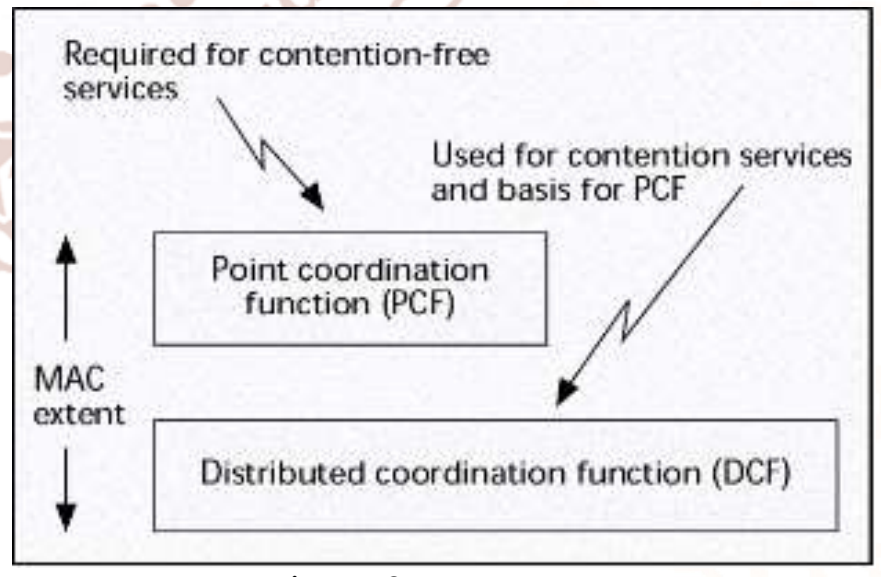

Figure 2 MAC Layer

\section{Mac in Ieee 802.11 In Multihop Scenario}

The IEEE 802.11 MAC is designed for wireless LANs. The requirements of multihop ad-hoc networks are more challenging than those of wireless LANs. We will investigate the operation of IEEE 802.11 MAC in centralized multihop ad-hoc networks. The 
terms station and node are used interchangeably throughout the thesis. Multihop cooperative wireless ad-hoc networks will be simply referred to as multihop networks

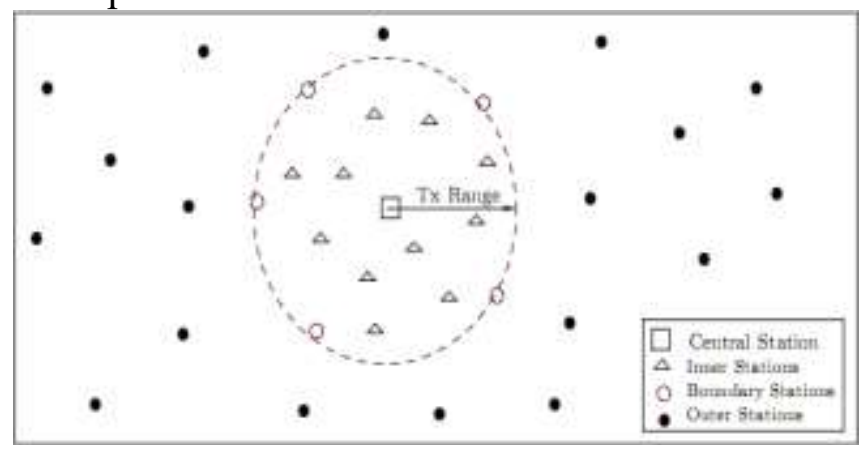

Figure 3: Multihop Scenario

Consider a multihop centralized scenario, as shown in the figure 3.For convenience; the stations inside the network are classified into following categories: Central station is the central controlling station. Most of the traffic in the network is directed towards it. Inner stations are within one hop boundary of the central station. Boundary stations are at one hop boundary of the central station. These stations act as relaying stations for the stations outside the reach of central node. Outer stations are outside the communication range of central node.

\section{IEEE 802.11 Operations in multihop networks}

The 802.11 MAC with DCF mode of operation is the simplest choice in multihop ad-hoc networks. The reason for the choice of DCF is that it does not require any prior infrastructure. Two or more stations can come together and form an BSS. This nature of DCF is very suitable for ad-hoc networks as the ad-hoc networks are simply formed by as set of stations coming together. In this section we discuss the operation of 802.11 MAC in multihop networks, especially centralized multihop ad-hoc networks In a centralized multihop network, as shown in Figure 4, the node density in central region is higher than in the outer region. Most of the traffic is directed toward the central node and boundary stations act as relaying stations. Therefore, the traffic near the central station and its one hop neighbors is very high. Since the DCF is a contention based distributed protocol, it performs badly in high load conditions. The poor performance of DCF is due to fact that the collisions increase as more and more stations try to access the medium at the same time. It is well known that the polling the most suitable choice for the polling MAC would be PCF mode of 802.11, as it is an extension of the DCF mode. Ebert ET. All [8] have shown that the PCF mode performs better than DCF when the number of stations in WLAN cell is very high. Therefore, we make the central node as Point Coordinator (PC), and it polls all the inner and boundary nodes during CFP period. This differs from conventional PCF operation in WLANs where PC resides within AP. The outer stations still perform DCF since the traffic in those regions is not high. The outer stations can send their data in contention period $(\mathrm{CP})$ as all the stations perform DCF during CP. We refer this combination of PCF and DCF as hybrid operation as shown in figure 4.

The hybrid operation seems to be an ideal choice in multihop networks, but it gives rise to following problems:

The stations that are polled by the Point Coordinator (PC) keep their NAV set during the CFP period, and therefore, cannot receive from outer stations. It can also be said that the boundary nodes become exposed to $\mathrm{PC}$.

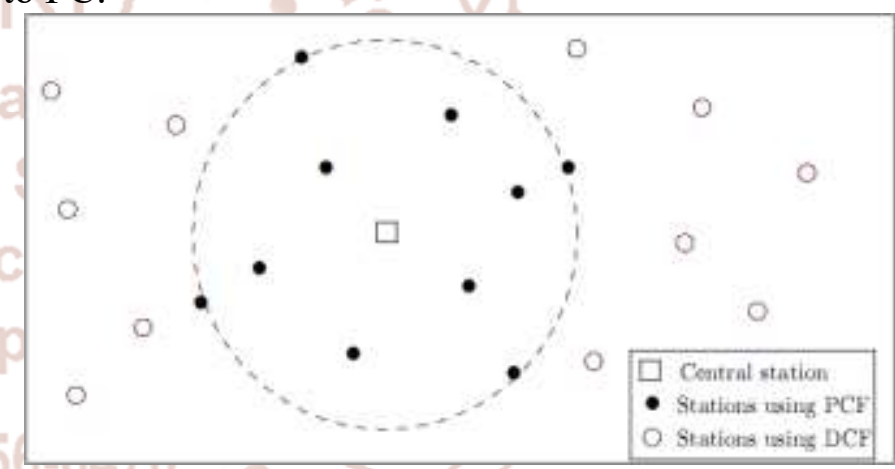

Figure 4 Hybrid PCF-DCF Operations

Outer stations become hidden to $\mathrm{PC}$, and vice versa, as there is no RTS/CTS exchange between PC and its one hop neighbors during CFP period.

\section{Problem Description}

Simple DCF is not suitable for centralized multihop network due to collisions at high traffic. A polling MAC (PCF) is required at the centre of the network to handle high traffic and reduce collision, but it gives rise to hidden and exposed node problems. The solution to both of these problems is provided by introducing dual Nodes at the boundary of the central node.

\section{DUAL MAC}

Due to these reasons we introduce Dual MAC,

1. The DCF does not work well in high load scenario.

2. In case of hybrid operation, the polling and NAV 
setting in PCF nodes cause exposed and hidden node problems, thereby decrease the throughput. To improve the throughput, boundary nodes should be able to receive date from outer nodes during the CFP period (NAV is set). For this the MAC should be able to receive even if its NAV is set. Also, transmissions from outer stations should not collide with that of PC at boundary stations. To address above problems, we propose to equip boundary stations with dual MAC. A dual node is a station which has two independent MACs each communicating on different logical channels. The two MACs are encapsulated inside the dual MAC. The logical channels could be FDMA or CDMA. Consider the boundary stations in Figures 4 and 5 that are equipped with dual MACs. One of the MACs uses the PCF and is termed as PCF MAC. The second MAC uses the DCF and is termed as DCF MAC. The PCF MAC communicates with the PC, and the DCF MAC communicates with the outer nodes. The exposed and hidden node problems in central region are eliminated as follows:

$>$ Boundary stations use the PCF and the DCF on different channels. Therefore, the transmission of outer node does not collide with that of PC, and vice versa.

$>$ The DCF MAC in the dual node can receive from outer nodes even when the NAV of PCF MAC is set during CFP period, thereby eliminating exposed node problem.

\section{Architecture of Dual MAC}

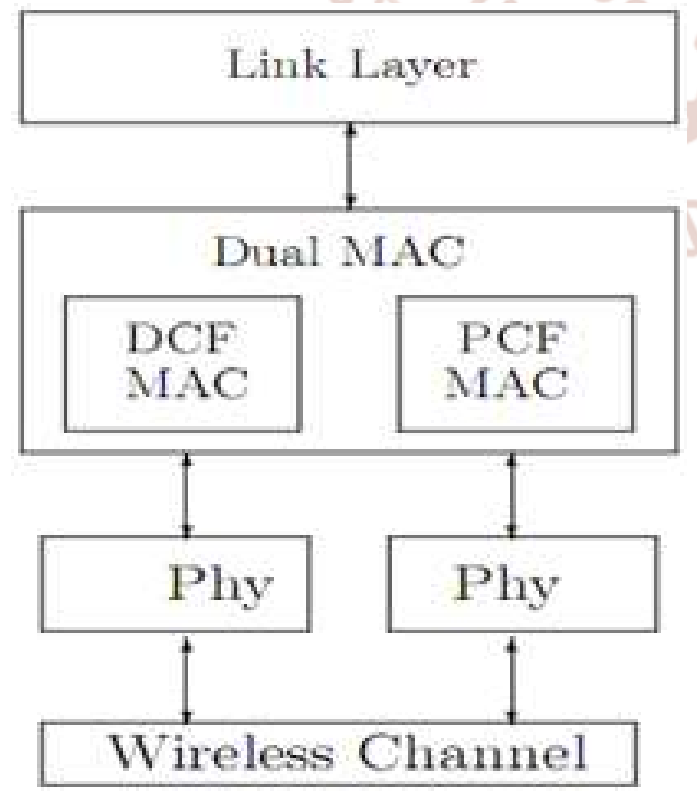

Figure 5: Architecture of Dual Mac Operation

A packet arriving from link layer is received by the dual MAC and handed over to the MAC at appropriate frequency. The link layer find out the MAC address of the next hop destination by using $\mathrm{ARP}$ and hands out the packet to the dual MAC layer along with the destination MAC address, In case dual MAC, the dual MAC also needs to know the channel of the destination station. This could be done either by ARP table maintaining information about the channel on which the destination stations is communication, or by maintaining a local list of stations on each channel. The dual MAC figures out the channel of the destination MAC and sends out the packet to the appropriate MAC. The broadcast packets like route discovery packets and ARP packets are sent to both the Macs. On receiving a packet from layer, the dual MAC simply hands it out to the link layer. The operation of the dual MAC is summarized in figure 6

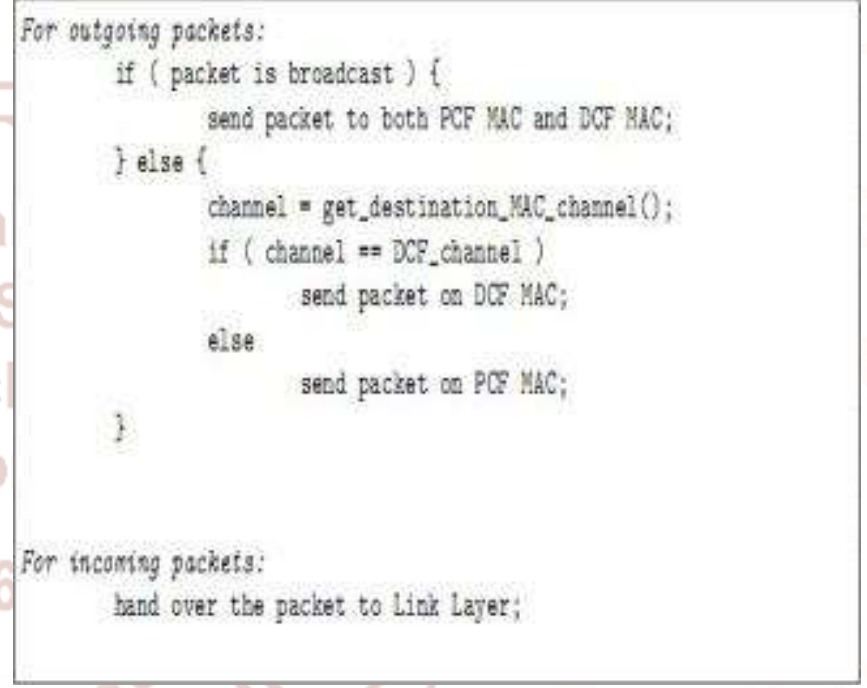

Figure 6 Operation of Dual MAC

4. RESULT \& DISCUSSION

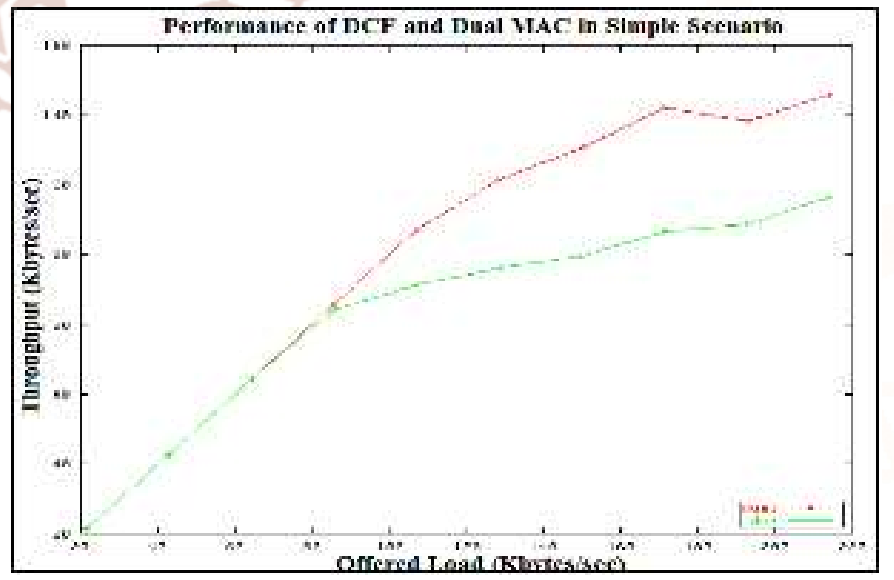

Figure 7 Throughput comparison of Dual MAC Vs DCF MAC in simple scenario 


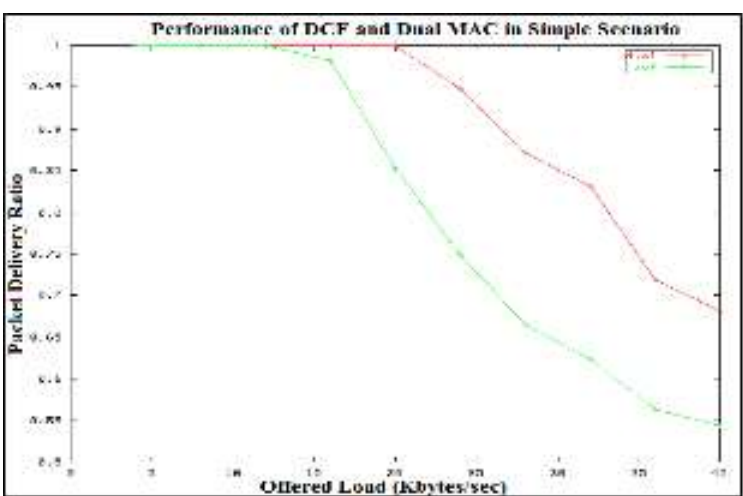

Figure 8 Packet Delivery Ratio for Dual MAC Vs DCF MAC in simple scenario

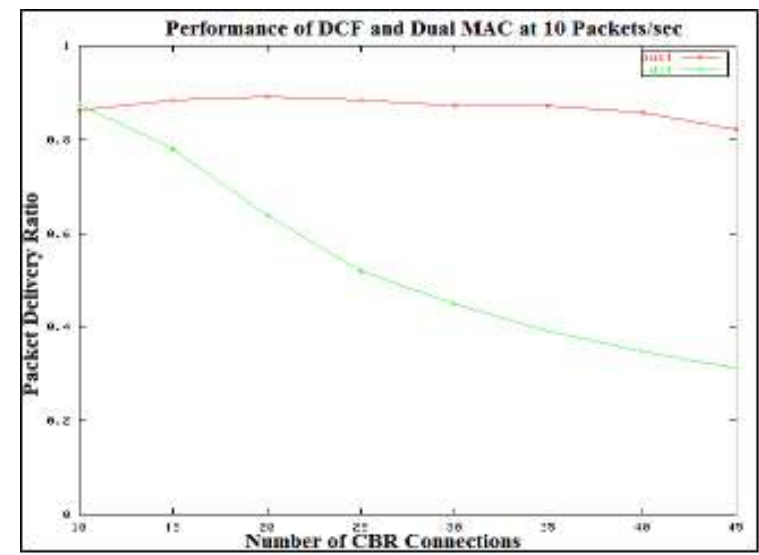

Figure 9 Dual MAC Vs DCF MAC at

10 packets / sec

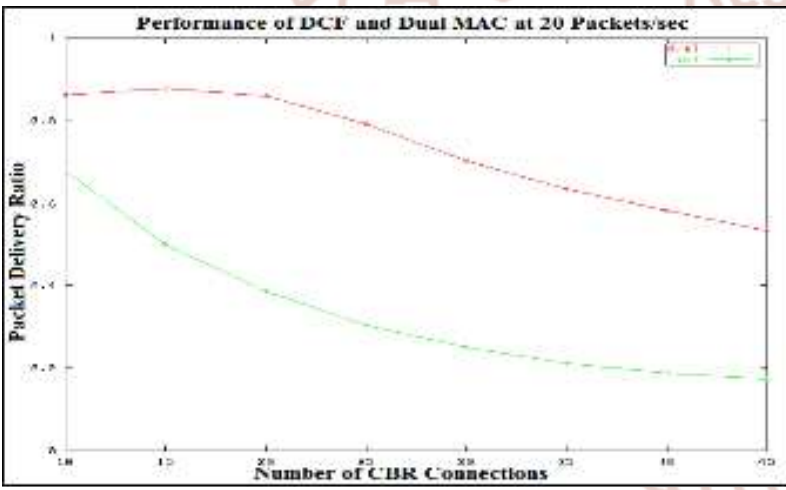

Figure 10 Dual MAC Vs DCF MAC at 20 packets / sec

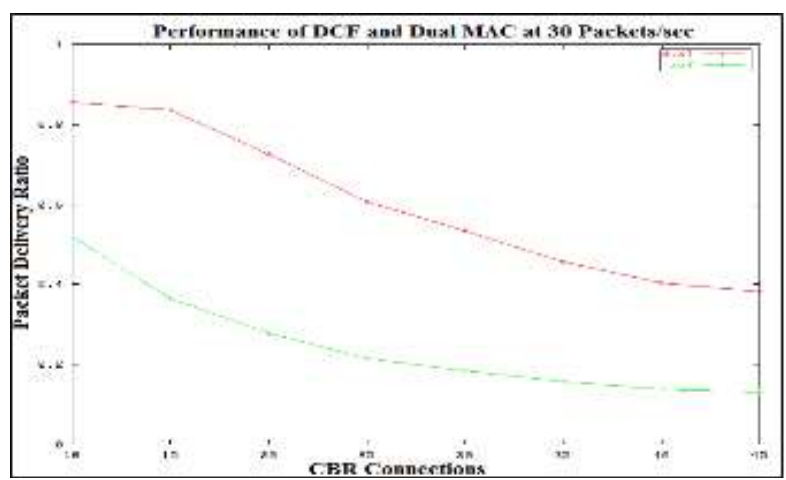

Figure 11 Dual MAC Vs DCF MAC at 30 packets / sec

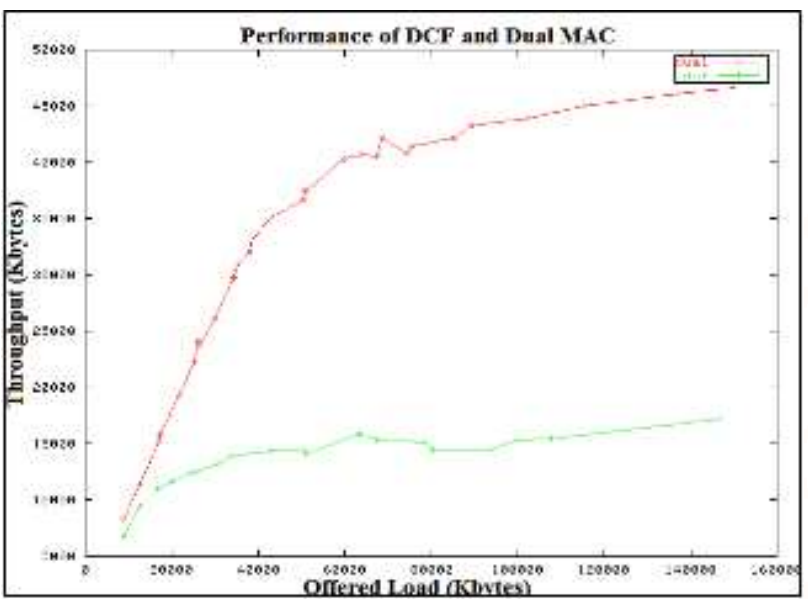

Figure 12 Dual MAC Vs DCF MAC - overall Throughput

\section{CONCLUSION}

The results show that the dual MAC performs reasonably better than the DCF access mechanism. However, the dual MAC requires two physical radios and two separate channels - one bound to PCF and another to DCF. We may however, note that the throughput gain is worth the cost of dual MACs since only few nodes need to be equipped with dual MAC.

The main focus of this thesis is to suggest a modification to the existing IEEE 802.11 MAC so as to make it suitable in multihop ad-hoc networks, especially in the real life centralized networks. The results presented in the thesis are applicable for static scenario, nevertheless, the dual MAC is expected to perform better even under mobile scenario. The effect of mobility on performance of dual MAC still remains to be seen.

\section{REFERENCES}

1. C. Perkins, Network Working Group, RFC 3220: IP Mobility Support for IPv4 http://www.ietf.org/rfc/rfc3220.txt.

2. Charles E. Perkins ET. all, Mobile Ad Hoc Networking Working Group, Ad hoc On-Demand Distance Vector (AODV) Routing, http://www.ietf.org/internet-drafts/draft-ietfmanet-aodv- 12.txt.

3. David B. Johnson, David A. Maltz, Yih-Chun Hu, and Jorjeta G. Jetcheva, IETF MANET Working Group, The Dynamic Source Routing Protocol for Mobile Ad Hoc Networks (DSR), http://www.ietf.org/internet-drafts/draft-ietfmanet-dsr-07.txt.

4. Charles E. Perkins, Pravin Bhagwat, Highly dynamic Destination-Sequenced Distance- Vector 
routing (DSDV) for mobile computers, ACM SIGCOMM Computer Communication Review, v.24 n.4, p.234-244, Oct. 1994.

5. IEEE Std. 802.11, Wireless LAN Media Access Control (MAC) and Physical Layer (PHY) Specifications, 1999.

6. Brian P. Crowe, I. Widjaja, J. Kim, P. Sakai, IEEE 802.11 Wireless Local Area Networks, IEEE Communications Magazine, September 1997.

7. Phil Karn, MACA - A New Channel Access Method for Packet Radio, ARRL/CRRL Amature Radio 9th Computer Networking Group paper 801.22/92-39, March, 1992.

8. Andreas Kopsel, Jean-Pierre Ebert, and Adam Wolisz, A Performance Comparision of Point and Distributed Coordination Function of an IEEE 802.11 WLAN in the presence of Real-Time Requirements, Proc. of 7th Intl. Workshop on Mobile Multimedia Communications (MoMuC2000), October 23-26, 2002.

9. Shugong $\mathrm{Xu}$, Tarek Saadawi Does IEEE 802.11 MAC Protocol Work Well in Multihop Wireless Ad Hoc Networks?, IEEE Communications Magazine, p.130-137, June 2001.

10. Neeraj Poojary, Srikanth V. Krishnamurthy, and Son Dao, Medium Access Control in a Network of Ad Hoc Mobile Nodes with Heterogeneous Power Capabilities, IEEE International Conference on Communications (ICC 2001), volume 3, p.872877, 2001.

11. J. Deng, and Z. J. Haas, Dual Busy Tone Multiple Access (DBTMA): A New Medium Access Control for Packet Radio Networks, IEEE ICUPC'98, Florence, Italy, October 5-9, 1998.
12. Nasipuri, and S. R. Das. A Multichannel CSMA protocol for multi-hop wireless networks, Proc. of IEEE Wireless Communications and Networking Conference (WCNC'99), September 1999.

13. Asis Nasipuri, and Samir R. Das, Multichannel CSMA with Signal Power-Based Channel Selection for Multihop Wireless Networks, Proceedings of the IEEE Fall Vehicular Technology Conference (VTC 2000), September 2000.

14. The VINT project, NS notes and documentation, editors: Kevin Fall and Kannan Varadhan, http://www.isi.edu/nsnam/ns.

15. Ad Kamerman and Leo Monte ban, Wave LANII: A High-Performance Wireless LAN for the unlicensed band, 1997.

16. Anurag Kumar Sridhar Iyer, Krishna Paul and Bhaskar Ramamurthy. Wifire: Medium access control (mac) and physical layer (phy) specifications, May 2006

17. LAN/MAN standards Committee. Part 11: Wireless lan medium access control (mac) and physical layer (phy) specifications. IEEE-SA Standards Board, June 2003.

18. LAN/MAN standards Committee, IEEE Microwave Theory, and Techniques Society. Air interface for fixed broadband wireless access systems. IEEE-SA Standards Board, June 2004.

19. Spin Documentation.

http://spinroot.com/spin/whatispin.html.

20. Bhaskaran Raman Pravin Bhagavat and Dheeraj Sanghi. Turning 802.11 inside-out.ACM SIGCOMM,Jan2004 